\title{
REVIEW ON APPLICATIONS OF PHOTOACOUSTIC SPECTROMETER
}

\author{
SAPNA $^{1} \&$ P. BHASKAR ${ }^{2}$ \\ ${ }^{1}$ Department of Instrumentation Technology, P.G. Centre, Gulbarga University, Raichur, Karnataka, India \\ ${ }^{2}$ Professor, Department of Instrumentation Technology, P.G. Centre, Gulbarga University, Raichur, Karnataka, India
}

\begin{abstract}
Photoacoustic (PA) effect, is basically generation of acoustic waves on irradiating certain substances by a modulated radiation source. Photoacoustic (PA) effect was discovered by A.G. Bell in 1881. Photoacoustic technique is a powerful tool for the determination of phase transitions in solids, liquids and gases compared to other conventional techniques. To explain the properties of the materials and to develop new materials, a basic understanding of phase transformation is required. PA signal information can be used to investigate the variations in the optical and thermal properties of the materials during phase transitions. The advantage of PA technique is its simplicity in the sample preparation, and its accuracy in the phase transition detection. The designing of photoacoustic spectrometers by using sophisticated systems, such as PC/Microprocessor/Microcontroller and DSP is reviewed.
\end{abstract}

KEYWORDS: Photoacoustic Spectrometer

Received: Apr 22, 2017; Accepted: May, 27, 2017; Published: Jul 31, 2017; Paper Id.: IJEEERAUG20177

\section{INTRODUCTION}

Photoacoustic spectroscopy is a widely used spectroscopic tool. The PA technique is a promising tool for measuring the thermal parameters such as thermal diffusivity, thermal conductivity and specific heat of gases, liquids and solids. Alexander G.Bell proposed spectrophone for the purpose of the absorption spectra of bodies in those portions of the spectrum that are invisible [1-2]. This instrument was used in his experiments for the transmission of sound without a cable connection. In the late 1960, the first applications of the Photoacoustic effect to trace gas monitoring were reported. The invention of the laser as an intense light source, the development of highly sensitive sound detectors (such as condenser microphones and pie zoelectric transducers), and the first comprehensive theoretical description of the photoacoustic effect in solids by Rosencwaig and Gersho: the socalled RG theory lead to the rediscovery of the Photoacoustic effect for analytical purposes [3]. A review is made on the significant developments in photoacoustic spectroscopy (PAS).

Photo thermal (PT) techniques are the absorption of light in a sample, with a change in its thermal state. This may be either a change of the temperature or other thermo dynamical parameter of the sample related to the temperature. Measurement of either the temperature, pressure or density change that occurs due to optical absorption is ultimately the basis for all PT spectroscopic methods. In contrast to conventional transmission spectroscopy, neither scattering nor reflected light contributes to the signal. This makes PT spectroscopy particularly attractive for absorption measurements in gaseous, liquid and solid media. The most straight forward detection scheme for a photoacoustic signal is the observation of the temperature change at the irradiated sample surface. The more common technique is the detection of the emitted thermal radiation from the sample surface, which represents the temperature distribution within the sample. If the temperature rise in the absorbing sample 
volume occurs faster than this volume can expand a local pressure increase (wave) is the consequence. This pressure wave can be considered as a sound signal. PT absorption measurements based on the detection of this sound wave are named Photoacoustic (PA) techniques [4]. PA signal information can be used to investigate the variations in the optical and thermal properties of the materials during phase transitions.

\section{PRINCIPLE}

Photoacoustic (PA) effect is basically generation of acoustic waves on irradiating certain substances by a modulated radiation source. Thus, when the sample is irradiated by modulated electromagnetic wave, absorption of photons (photo energy) generates excited internal energy levels. All or part of the absorbed photon energy is then transformed into heat (thermal energy) through non-radiative relaxation processes in the sample [5]. The block diagram of Photoacoustic Spectrometer is as shown in Figure 1

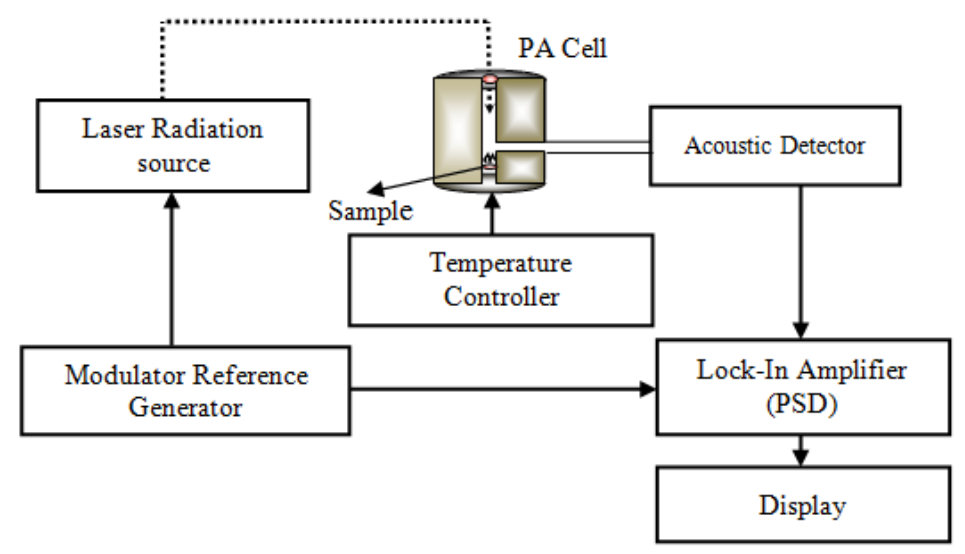

Figure 1: Block Diagram of Photoacoustic Spectrometer

Photoacoustic spectrometers (PAS) can be broadly classified into two categories

Photoacoustic Spectrometer for spectral studies that employ a variable wavelength radiation

Photoacoustic Spectrometer, which employs a fixed wavelength laser

Several investigators have improved the basic PAS technique by incorporating several modifications in the cell design, radiation sources, detectors, data acquisition and processing systems etc,.

\section{ANALOG TECHNIQUES}

S.Lakshminarayana et al [6] fabricated a compact Photoacoustic spectrometer (PAS) for the determination of phase transitions in liquid crystals (LCs). A Resonance Photoacoustic cell was used. The sample was placed in a small aluminium pan and was kept inside the resonance cavity of the PA cell. A commercial temperature controller was used to control the temperature; a heating cartridge was inserted through a hole in the PA resonance cell. The temperature was measured with a digital thermometer, at intervals of $0.5^{\circ} \mathrm{C}$, The PA signal picked up by a microphone was amplified by a preamplifier LA3161 and was connected to the input channel of the lock-in amplifier. The modulating frequency of the laser diode was the reference signal to the lock-in amplifier. By placing a glass plate coated with carbon soot in the cavity and observing the PA signal, the performance of the instrument was checked. The PA signal was detected over a wide thermal range from $30^{\circ} \mathrm{C}$ to $150^{\circ} \mathrm{C}$. The instrument could detect the phase transition of p-pentyloxy benzoic acid (5OBA) 
at $121.76^{\circ} \mathrm{C}, 151.4^{\circ} \mathrm{C}$ and $150.4^{\circ} \mathrm{C}, 113.6^{\circ} \mathrm{C}$, simultaneously both during heating and cooling cycles, this indicates the suitability of this setup for PA studies of solids, liquids and liquid crystals, over a wide a temperature range and with a high degree of accuracy.

Similarly J.,Fernandz etal, designed a resonant photoacoustic cell for a wide temperature range $\left(200^{\circ}-500^{\circ} \mathrm{K}\right)$, for the study of phase transitions in solids, and proved that photoacoustics measurements are highly sensitive for the study of phase transitions in solids as well as the behavior of the thermal conductivity. The complete experimental arrangement is shown in figure 2. AHe-Ne $4 \mathrm{mWlaser}$ was used as the light source the radiation beam was chopped at $108 \mathrm{~Hz}$ by an electromechanical chopper. An electret condenser microphone together with a pre-amplifier and an auto phase lock-in amplifier were used for the PA signal detection. The PA cell calibration was carried out with black carbon of known thermal properties [7].

J.C. Kapil et al [8] designed and fabricated a low-temperature resonant photoacoustic cell, based on Helmholtz resonator model, for the study of samples like ice or snow. The results obtained are in good agreement with those predicted by Rosencwaig-Gersho (R-G)' theory. And, showed that nondestructive treatment of a sample in photoacousticspectroscopy is helpful in the study of thermal and optical properties of ice and snow.

C.K.N. Patel focused on the recent use of broadly tunable single frequency high power room temperature quantum cascade lasers (QCL) for the detection of the CWAs and explosives. He developed room temperature QCLs that produce continuously tunable single frequency $\mathrm{CW}$ power output in excess of $300 \mathrm{~mW}$ at wavelengths covering $5 \mu \mathrm{m}$ to $12 \mu \mathrm{m}$ (using external grating cavity geometry). He presented data that showed a CWA detection capability, and also showed the capability of detecting a variety of explosives at a ppb level, with very low false alarm rates. The capability of detecting homemade explosives such as triacetone triperoxide and its liquid precursor, acetone which is a common household liquid were the explosives, which were demonstrated. [9].

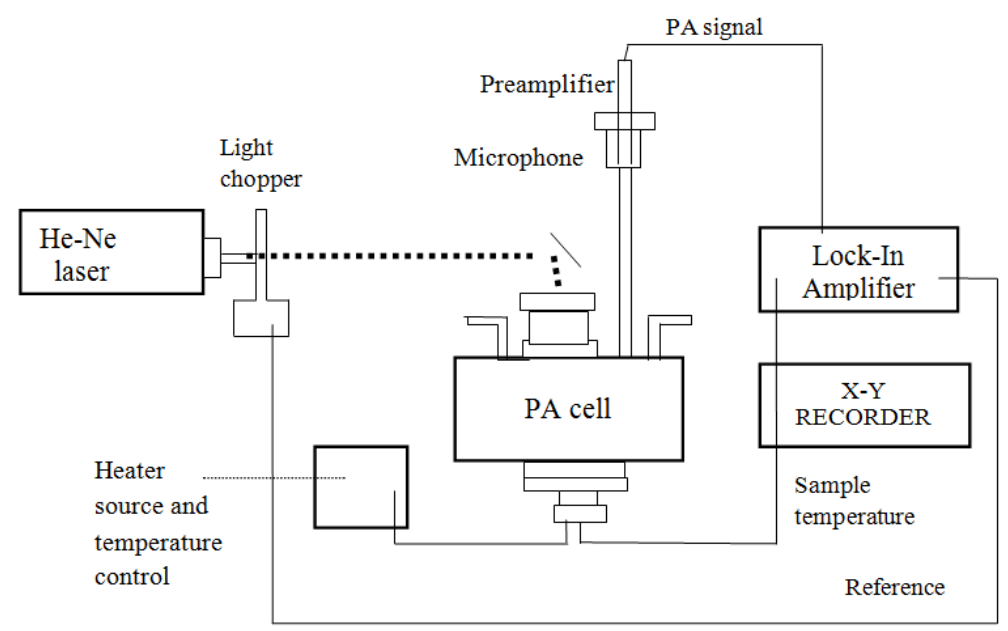

Figure 2: Complete Experimental Arrangement of Photoacoustic Spectrometer

\section{DIGITAL TECHNIQUES}

The PAS designed using analog techniques, inspite of having wide frequency of operations \& analysis; they are still associated with many problems like temperature drift, ageing effects, nonlinearities in measurement etc. hence digital techniques were employed. 


\section{Microprocessor Based Photoacoustic Spectrometer}

A.H.G. El-Dhaher et al, [10] developed a microprocessor-based system to enable on-line processing of data transferred from a photoacoustic spectrometer via a direct interface. The developed system uses the data transmission port of the spectrometer and does not require any modification of the original instrument. The study showed that data analysis requires comparison of several spectra, which was easy with the new designed system. Previously, it was not possible to store a number of spectra and data acquisition took a long time. The photoacoustic spectrometer used in this experiment was PAR Model 6001. A 1000-W xenon lamp was used as a source of excitation. Air was used as a coupling medium between the microphone and the sample. The modulation frequency of the xenon lamp was $40 \mathrm{~Hz}$. Commercial polyurethane rigid foam based on methyl is (4-phenyl diisocyanate) (MPBI) was used in this experiment. Hence, the system allowed the user to transfer data from the photoacoustic spectrometer to the system. The interface did not require any hardware or software changes to the original instrument. Data received by the developed system were identified, under software control, and stored in memory for further processing. A wide range of data processing, for both calculation and graphical presentation, was offered to the user. Up to eight different sets of data representing eight different runs can be stored and processed by the system. All system operations were done through a graphic/alphanumeric terminal equipped with video screen and keyboard. The video screen was used to display system current status and output results. The system was commanded by entering short answers to questions displayed by the system; hence no programming knowledge was required. All this is achieved in an easy-to-use, portable and moderate-cost system. Figure 3 shows the block diagram of the microprocessor based Photoacoustic spectrometer.

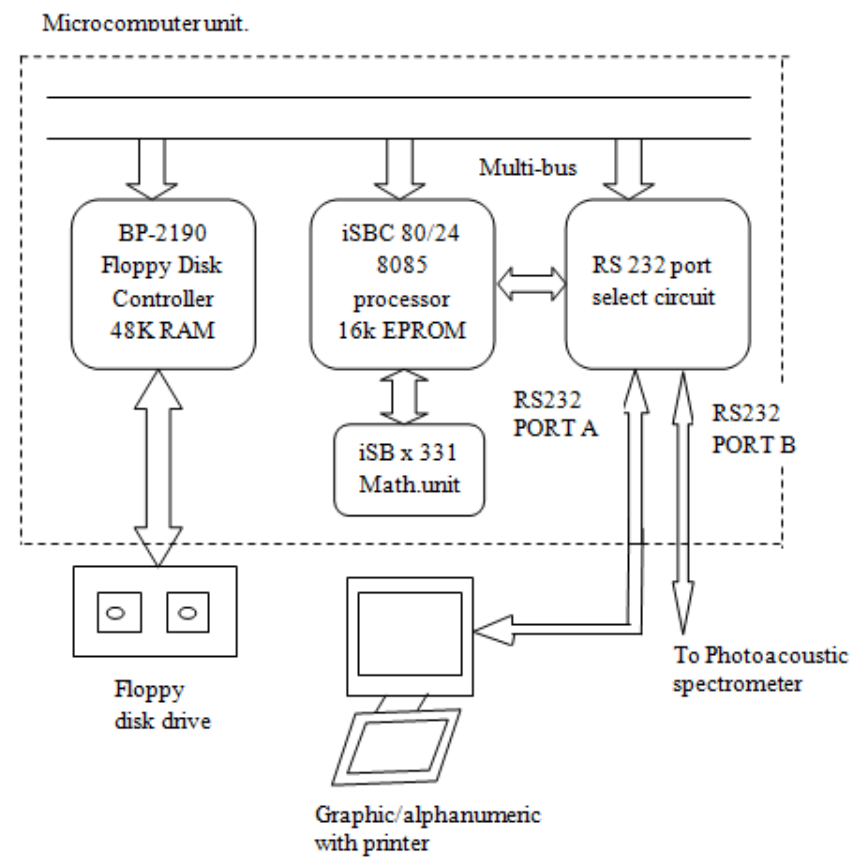

Figure 3: Block Diagram of the Microprocessor Based Photoacoustic Spectrometer

\section{P.C Based Photoacoustic Spectrometer}

The interfacing of photoacoustic spectrometer (PAS) to an IBM-PC via the RS232 communication link was reported by M.E. Abu-Zeid et al [11].Turbo Basic 1.1 and Turbo Pascal 3.0 were the programming languages that were used to transmit and process the data, that was produced by the PAS system. Many new graphical forms were produced by 
the software, to display the PAS data. By using some of the options such as plotting quadrature vs. in phase and PAS signal vs. wave number or locating major peaks, some thin films' parameters were computed accurately. To calculate thickness of non-conductive thin films electrodeposited on conducting substrates, a simulation equation for the PAS signal was developed and tested. And, this equation could also be used to calculate the refractive indices of the thin films, if the refractive indices of the substrates are known and vice versa.

K. Jeyadheepan et al, developed a photoacoustic spectrometer and the instrument control programs, using Agilent VEE Pro virtual engineering software [12]. H. Benamrani et al [13] analyzed the optical properties of silicon single crystals at different frequencies between 25 and $312 \mathrm{~Hz}$ by using a high resolution fully automated photoacoustic spectrometer (PAS) of the gas-microphone type in the photon energy region $0.8-1.6 \mathrm{eV}$. A comparison was carried out between the results obtained using the two techniques in determining the absorption coefficient and the gap energy.

The design and development of computer based instrumentation system for photoacoustic studies was described by P. Bhaskar [14]. This instrument was employed for phase transition studies in liquid crystals, with special reference to nO.m compounds and benzoic acids and hydrogen bonded complexes. The Block diagram of PC based photoacoustic Spectrometer is as shown in Figure 4. A modulated laser beam $(20 \mathrm{~mW})$ was used as a radiation source; The acoustic disturbances generated in the sample cell were detected by the electret microphone. The microphone output was amplified by high gain pre-amplifier designed using LA3161 dual operational amplifier. The output of the pre-amplifier was passed through the narrow band-pass filter, which enhances signal-to-noise $(\mathrm{S} / \mathrm{N})$ ratio of the signal. Finally, the output of the band-pass filter was given to the computer based lock-in amplifier; the later recovers the photoacoustic signal obscured by the noise. The temperature controller was used to control the temperature of the photoacoustic cell to the desired temperature, which enables to study the sample characteristics as a function of temperature.

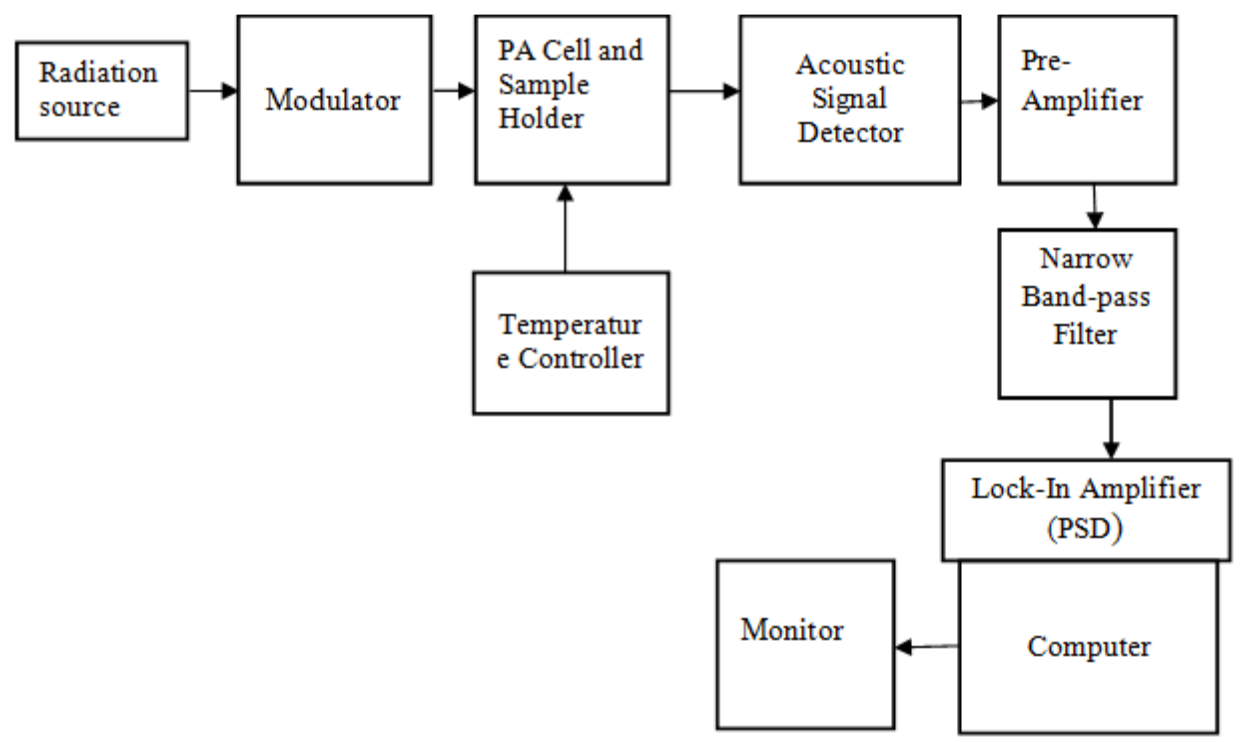

Figure 4: Block Diagram of PC Based Photoacoustic Spectrometer

\section{Microcontroller and DSP Based Photoacoustic Spectrometer}

In order to reduce the size and cost of the device, the mechanical and optical components of the equipment had to be replaced by electronics. The electronic part of the system was strongly reduced by intensive implementation of digital signal processing techniques instead of traditional hardware solutions. Virtually, in any kind of research, it is usually 
required that multiple measurement conditions can be changed independently, so that, it is possible to evaluate their influence on measurement results and to find optimal conditions for the measurements. It can be easily noticed from the literature, in the field of photo acoustics that such an approach usually results in huge, complex and very expensive photo acoustics systems, despite from the fact that, due to constant and significant progress in technology, at least standard components of photo acoustics experimental setups should decrease in size and price. It should be emphasized that even improved designs of photo acoustics set-ups that are based on microcontrollers and modern technology do not make optimal use of electronics and digital signal processing techniques [15-19].

Hence, a truly miniature system for data acquisition and control, dedicated for photo acoustics measurements was described by Tomasz Starecki [20]. Due to intensive use of electronics and digital signal processing, most of the inconvenient elements of photoacoustic setups, like mechanical chopper or lock-in amplifier, were eliminated. Figure 5 shows the block diagram of the system. The analog part of the device consists of blocks, in which the photoacoustic signal is amplified and converted to digital samples. The digital part of the device was separated from the analog part with optocouplers.

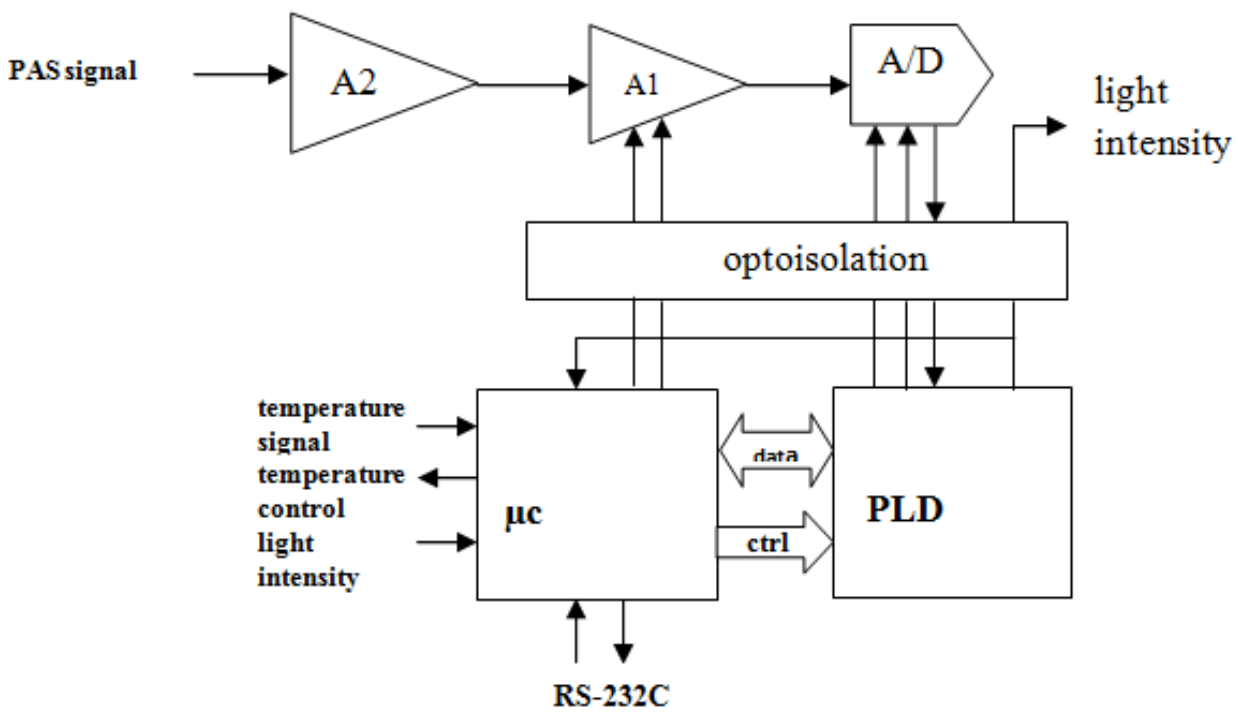

Figure 5: Block Diagram of the Microcontroller Based PAS

P. Bhaskar et al, described the design and development of microcontroller based photoacoustic spectrometer (PAS). A Helmholtz resonator type of photoacoustic (PA) cell was designed for the present study to measure the sample behavior as a function of temperature. An electret microphone was employed to achieve high signal to noise ratio. The microphone compartment was isolated from the sample compartment to avoid the heating effect of the resistive electric microphone, which detects the photo acoustic signal. A two stage preamplifier and tunable band-pass filter was designed. Microcontroller has been used to acquire and process the signal from the photoacoustic cell. The frequency response of the cell was studied with carbon-black as a sample. The system is very compact and easy to carry the experiments [21]. Figure 6 shows the block diagram of the complete system. 


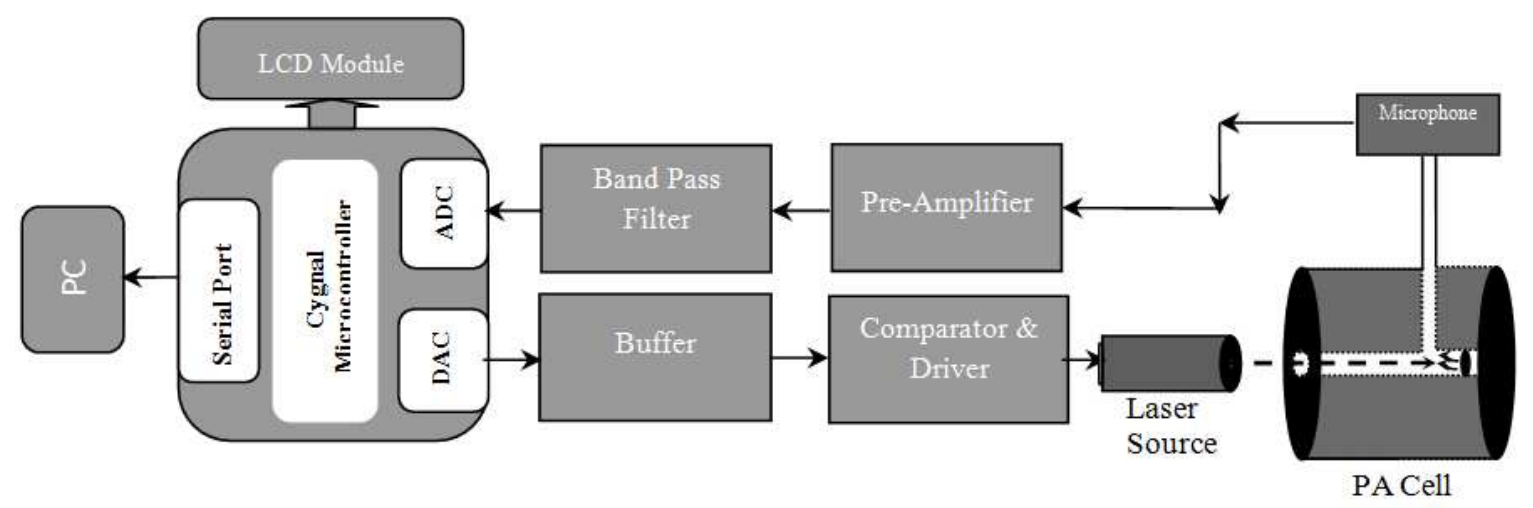

Figure 6: Block Diagram of Microcontroller Based Photoacoustic Spectrometer

\section{CONCLUSIONS}

From the literature survey, it is found that various authors proposed analog type of photoacoustic spectrometers and they have studied its wide applications such as determination of phase transitions in liquid crystals (LCs). Where PA signal information was used to investigate the variations in the optical and thermal properties of the materials during phase transitions, they proved that photo acoustics measurements are highly sensitive for the study of phase transitions in solids as well as the behavior of the thermal conductivity. The study of samples such as water, ice, glass, plexi-glass, polycarbonate etc., was carried out by the designed photoacoustic cell for low temperature ranges. Detection of the CWAs and explosives such as triacetone triperoxide and its liquid precursor, acetone which is a common household liquid could be done. In the case of digital type of photoacoustic spectrometer, a microprocessor-based system to enable on-line processing of data transferred from a photoacoustic spectrometer via a direct interface was developed. The developed system used the data transmission port of the spectrometer and does not require any modification of the original instrument. All system operations were done through a graphic/alphanumeric terminal equipped with video screen and keyboard. The video screen was used to display system current status and output results. The system was commanded by entering short answers to questions displayed by the system; hence no programming knowledge was required. All this is achieved in an easy-to-use, portable and moderate-cost system.

To calculate thickness of non-conductive thin films, it was electrodeposited on conducting substrates. A simulation equation for the PAS signal was developed and tested, which could also be used to calculate the refractive indices of the thin films, if the refractive indices of the substrates are known and vice versa. Some research found that a photoacoustic spectrometer and the instrument control programs, using Agilent VEE Pro virtual engineering software were developed. The optical properties of silicon single crystals at different frequencies between 25 and $312 \mathrm{~Hz}$, by using a high resolution fully automated photoacoustic spectrometer (PAS) were analyzed.

The miniature system for data acquisition and control, dedicated for photo acoustics measurements was described. Most of the inconvenient elements of photoacoustic setups, like mechanical chopper or lock-in amplifier, were eliminated due to intensive use of electronics and digital signal processing.

\section{REFERENCES}

1. Bell A. G. "Photoacoustic imaging and spectroscopy,”American Journal ofScience 20:305. (1880).

2. Bell A G "Laser photoacoustic: A novel method for ethylene determination," PhilMag J Sci 11:510. (1881). 
3. Rosencwaig A, Gersho, “ ATheory of Photoacoustic effects in solid,” J. Apply. Phys. 47:64. (1976)

4. C.Haisch and R.Niessner "Light and Sound-Photoacoustic spectroscopy,” Specctroscopy Europe,pp.10-15, 2002.

5. Rosencwaig, A.,“Photo-acoustic Spectroscopy of Solids,”Rev. Sci. Instrm. 48, 1133, 1977.

6. S. Lakshminarayana, K. Gouthami, V. G. K. M. Pisipati, N. V. S. Rao \& G. Venkata Rao, "Accurate Method for the Determination of Phase Transitions in Liquid Crystals with Photoacoustic Technique," pp. 1520-1528.

7. J Fernhndez, J Etxebarria, M J Tello and A L6pez Echarri, "A wide temperature range photoacoustic cell for the study of phase transitions in solids: an application to the ferroelectric-incommensurate ( $(\mathrm{CH}),), \mathrm{CoCl}$," J. Phys. D: Appl. Phys, no. 16, pp. 269-274 (1983)

8. J.C. Kapil, S.K. Joshi, and A.K. Rai, "Fabrication of a Resonant Photoacoustic Cell for Samples Study," Defence Science Journal, vol. 54, no. 2, pp. 209-218, April 2004

9. C.K.N. Patel, "Laser photoacoustic spectroscopy helps fight terrorism: High sensitivity detection of chemical Warfare Agent and explosives," Eur. Phys. Journal, Special Topics 153, pp. 1-18, (2008).

10. A.H.G. El-Dhaher, H.A. Kaouri, M.E. Abu-Zeid, M.S. Adili, "An interactive microprocessor-based system for the processing of photoacoustic spectrometer data,"

11. M.E. Abu-Zeid, H.A. Kordia, Y.A. Youssef, "An interactive implementation of IBM-PC for processing and simulation of photoacoustic spectrometer data,” Journal of Microcomputer Applications, vol.7, no.3-4, pp. 203-215, October 1984,

12. K. Jeyadheepan, P. Palanichamy, P. Kalyanasundaram, M. Jayaprakasam, C. Sanjeeviraja,K. Ramachandran, "Automation of photoacoustic spectrometer using VEE Pro software," Measurement, vol. 43, pp. 1336-1344, (2010)

13. H. Benamrani, F.Z. Satour, A. ZegadiA. Zouaoui, " Photoacoustic spectroscopy analysis of silicon crystals," Journal of Luminescencevol. 132, no. 2, pp. 305-312, February 2012

14. P. Bhaskar, "Design And Development Of Computer Based Instrumentation System For Photoacoustic Studies" thesis submitted to Sri Krishnadevaraya University Anantapur - 515003 (A.P) India

15. S. L. Firebaugh, K.F. Jensen, and M.A. Schmidt, "Miniaturization and integration of photoacoustic detection”, J.Appl. Phys.92, pp. 1555-1563, 2002.

16. G.Santiago, V.Slezak, and A.L. Peuriot, “Resonnant photoacoustic gas sensing by PC-based audio detection”, Appl. Phys. B77, pp.463-465, 2003

17. K.Song, H.K.Cha, V.A.Kapitanov, Yu.N.Ponomariev, A.P.Rostov, D.Courtis, B.Parvitte, and V.Zeninari "Differential Helmholtz resonant photoacoustic cell for spectroscopy and gas analysis with room-temperature diode lasers", Appl.Phys. B75, pp. 215227, 2002.

18. Z. Bozoki, A. Mohacsci, G.Szabo, Z.Bor, M.Erdelyi, W.Chen, and F.K.Tittel, "Near-infrared diode laser based spectroscopic detection of ammonia: a comparative study of photoacoustic and direct optical absorption methods", Appl. Spectroscopy56 pp. 715-719, 2002.

19. Z. Bozoki, J.Sneider, Z. Gingl, A.Mohacsi, M. Szakall, Z. Bor, and G.Szabo, “ A high-sensitivity, near-infrared tunable-diodelaser-based photoacoustic water-vapour-detection system for automated operations”, Meas. Sci. Technol., 10, pp.999$1003,1999$.

20. Tomasz Starecki, Marcin Grajda "Low cost miniature data acquisition and control system for photoacoustic experiments., Photonics Applications in Astronomy, Communications, Industry, and High-Energy Physics Experiments IV.,pp-61592O- 
$615920-8,2006$

21. P. Bhaskar, Immanuel J., and Bhagyajyoti "Design and Development of Microcontroller based Photoacoustic Spectrometer," Sensors and Transducers, vol.141 no.6., pp.26-34

22. David L. Drapcho, Raul Curbelo, Eric Y. Jiang, Richard A. Crocombe, and William J. McCarthy, "Digital Signal Processing for Step-Scan Fourier Transform Infrared Photoacoustic Spectroscopy,” Applied Spectroscopy, vol. 51, no. 4, pp. 453-460, (1997)

\section{BIOGRAPHY}

Sapna completed her M.Sc. and M.Phil in Instrumentation Technology, from Gulbarga University. Presently, she is pursuing Ph.D. under the guidance of Dr. P. Bhaskar, Professor, Department of Instrumentation Technology, Gulbarga University Post Graduate Centre, Raichur. She has published 3 papers in journals of national/international repute and presented 2 papers in national/ international conferences.

P. Bhaskar received the M.Sc., M.Phil., and Ph.D. degrees in Instrumentation from Sri Krishnadevaraya University, Anantapur, AP, India, Currently, he is a Professor in the Department of Instrumentation Technology at Gulbarga University Post Graduate Centre, Raichur, KA, India. He has published more than 53 papers in journals of national/international repute and presented over 22 papers in national/ international conferences. He has also authored a book on 8051 microcontroller. His research interests include Scientific Instrumentation, Embedded Systems, and Intelligent Control Systems. He is a member of the Instrument Society of India. 
